\title{
Comparison between euglycemic hyperinsulinemic clamp and surrogate indices of insulin sensitivity in children with growth hormone deficiency
}

\author{
Alessandro Ciresi, Valentina Guarnotta, Giuseppe Pizzolanti, Carla Giordano* \\ Section of Endocrinology, Biomedical Department of Internal and Specialist Medicine (DIBIMIS), University of Palermo, Piazza delle Cliniche 2, 90127 Palermo, Italy
}

\section{A R T I C L E I N F O}

\section{Keywords:}

Growth hormone deficiency

Insulin sensitivity

Hyperinsulinemic clamp

\begin{abstract}
A B S T R A C T
Objective: Data about the impact of growth hormone treatment (GHT) on insulin sensitivity in children are quite controversial, due to the different surrogate indices that have been used.

Design: We evaluated insulin sensitivity through the euglycemic hyperinsulinemic clamp, considered the gold standard technique, in 23 children affected by growth hormone deficiency (GHD) at baseline and after 12 months of GHT and in 12 controls with short stature at baseline, and we compared the clamp-derived index (M-value) with the most commonly used surrogate index of insulin sensitivity, as ISI Matsuda, and with circulating plasma markers of insulin sensitivity, as adiponectin and resistin levels.

Results: At baseline, no significant difference in all metabolic parameters between GHD children and control subjects was found. After 12 months of GHT, GHD children showed a significant increase in fasting insulin $(p<0.001)$ and resistin $(p=0.028)$ and a decrease in ISI Matsuda $(p<0.001)$ and M-value $(\mathrm{p}<0.001)$, without significant change in fasting glucose, HbA1c and adiponectin. In GHD children, M-value showed a significant but weak correlation with ISI Matsuda (rho $0.418, p=0.047$ ) at baseline, while no correlation with other parameters was found. After 12 months of GHT, M-value did not show any significant correlation with any other metabolic parameter analyzed.

Conclusions: This study highlights the limit of the evaluation of insulin sensitivity performed through surrogate indices or circulating markers, which may lead to controversial data and do not correlate with the gold standard technique to evaluate insulin sensitivity.
\end{abstract}

\section{Introduction}

It is widely known that growth hormone (GH), in addition to its auxological effects, exerts an anti-insulin effect which can potentially induce changes in carbohydrate metabolism [1]. GH treatment (GHT) is associated with a tendency toward insulin resistance and a number of clinical indices have been used in children affected by GHD to assess the impact of GHT on insulin sensitivity, with various and conflicting results. For these reasons, a reliable assessment of insulin sensitivity in a population potentially at risk of impaired insulin sensitivity, like GHD patients during GHT, is mandatory. Unfortunately, most of the indices routinely used in clinical practice are derived from fasting glucose and insulin levels and have not always proved to be to be reliable measures of insulin sensitivity in healthy children [2]. Indeed, the gold standard for assessing insulin sensitivity is euglycemic hyperinsulinemic clamp [3] and Schwarts et al. demonstrated that most commonly used indices of insulin sensitivity are not widely correlated with the clamp [2]. Similarly, in a previous study including a small cohort of GHD children we demonstrated a lack of correlation between the clamp-derived Mvalue and other surrogate indices of insulin sensitivity, like Homa-IR or QUICKI, which did not represent reliable indices of the insulin sensitivity degree [4].

However, clamp is an invasive and expensive procedure and it is difficult to apply it routinely in clinical practice, especially in a large number of children. Consequently, reliable data on insulin sensitivity derived by clamp in GHD children are very scarce.

As plasma markers of insulin sensitivity, the circulating levels of some adipokines, such as adiponectin and resistin, have been evaluated in GHD but with very discordant data across the studies. In addition, no data about the relationship among adipokines and clamp are available in GHD children. Therefore, the reliability of adipokine levels is not entirely known.

The aim of this study was to use the hyperinsulinemic clamp to evaluate the impact of GHT on insulin-stimulated glucose utilization in a group of children affected by GHD and to compare the clamp-derived index with the most commonly used surrogate indices and plasma

\footnotetext{
Abbreviations: GH, growth hormone; GHT, growth hormone treatment; GHD, growth hormone deficiency

* Corresponding author.

E-mail address: a.ciresi@virgilio.it (C. Giordano).
} 
markers of insulin sensitivity.

\section{Materials and methods}

We prospectively studied 23 prepubertal children (15 M, $8 \mathrm{~F}$, mean age $8.4 \pm 1.0$ years; range 6.1-9.9) with isolated idiopathic GHD consecutively admitted to the Section of Endocrinology of the University of Palermo during the years 2014-2015 and treated with GH for at least 12 months and never investigated before in other clinical studies. Twelve prepubertal healthy subjects with short stature $(7 \mathrm{M}$, $5 \mathrm{~F}$ ) matched for age (mean age $7.8 \pm 0.9$ years; range 5.6-9.4) were recruited among children referred for assessment of short stature as a control group at baseline. All children were in the first stage of sexual development during the full observation period to avoid any interference of the onset of puberty with metabolic parameters. Similarly, children with multiple pituitary hormone deficiencies were excluded from the study to avoid any potential interference of untreated hormonal deficiency or of other hormonal replacement therapy on metabolic data.

GHD was diagnosed by the auxological and biochemical criteria of the GH Research Society [5]. As auxological data we considered height and growth velocity 1 year before diagnosis, while we considered a bone age delay, estimated from an X-ray of the left wrist and hand and evaluated according to the methods of Greulich and Pyle, of at least 1 year with respect to the chronological age [6]. Biochemically, GHD was demonstrated by failure of GH to respond to an arginine and glucagon stimulation test, performed on two different days. Specifically, GH peak was below $7 \mu \mathrm{g} / 1$ in 3 children, below $6 \mu \mathrm{g} / 1$ in 6 children, below $5 \mu \mathrm{g} / \mathrm{l}$ in 9 children and below $3 \mu \mathrm{g} / \mathrm{l}$ in 5 children. Since the classification of GHD in moderate or severe is quite arbitrary, we considered a GH peak below $8 \mu \mathrm{g} / \mathrm{l}$ as diagnostic for overt GHD, in line with the criteria of appropriateness of use and reimbursement of GHT in children according to the Italian Medicines Agency (AIFA). Neuroimaging of the hypothalamic-pituitary region, in line with our protocol, was performed in children with more severe GHD, like those with GH peak $\leq 3 \mu \mathrm{g} / \mathrm{l}$ during both tests ( 5 children) and showed no pituitary alterations.

The patients received GH once daily at bedtime. During the entire follow-up, insulin-like growth factor (IGF)-I levels allowed us to determine the GH dose. Specifically, the initial daily dose of GH was $0.025 \mathrm{mg} / \mathrm{kg}$, increased to $0.027 \mathrm{mg} / \mathrm{kg}$ from monthS 6 to 12 .

\subsection{Study protocol}

In all GHD patients the auxological and metabolic evaluation was performed at baseline and after 12 months of GH treatment, while in controls these evaluations were only performed at baseline. In all children we measured body height, body mass index (BMI) and waist circumference (WC). A blood sample was drawn after an overnight fast for the measurement of glucose, insulin, Hemoglobin A1c (HbA1c) and IGF-I. This sample served as the baseline for the oral glucose tolerance test (OGTT). Blood samples were collected every $30 \mathrm{~min}$ for $120 \mathrm{~min}$ for glucose and insulin measurements.

As surrogate estimates of insulin sensitivity we used the insulin sensitivity index, a composite index derived from the OGTT and validated by Matsuda and DeFronzo (ISI-Matsuda) [7].

On a different day, as a gold standard measurement of insulin sensitivity we performed the euglycemic hyperinsulinemic clamp. As per our protocol, one catheter was placed in a vein on the forearm for administration of insulin and glucose and the second catheter was placed in retrograde direction in an antecubital vein of the contralateral heated forearm for obtaining arterialized venous blood, using an airheated box at approximately $55^{\circ} \mathrm{C}$. The clamp was performed under standard conditions, i.e. the plasma insulin concentration was acutely raised with insulin priming $\left(0-3 \mathrm{~min}: 113.6 \mathrm{mU} / \mathrm{m}^{2}, 3-6 \mathrm{~min}\right.$ : $80.2 \mathrm{mU} / \mathrm{m}^{2}, 7-10 \mathrm{~min}: 50.4 \mathrm{mU} / \mathrm{m}^{2}$ of body surface area) for the first
$10 \mathrm{~min}$ of the test and maintained by continuous infusion of insulin infusion ( $40 \mathrm{mU} / \mathrm{m}^{2}$ for the remaining $110 \mathrm{~min}$ ). The rate of peripheral glucose utilization ( $\mathrm{M}$ value) was calculated by dividing the glucose amount infused during the last 40 min by body weight measured in kilograms $(\mathrm{mg} / \mathrm{kg} / \mathrm{min})$. The plasma glucose concentration was held constant at basal levels by variable glucose infusion and under the steady-state conditions of euglycemia the glucose infusion rate equaled glucose uptake by all the tissues in the body and it was therefore considered a measure of tissue sensitivity to exogenous insulin [3].

Multiple pituitary hormone deficiency at baseline was excluded by the evaluation of normal basal cortisol levels (which were $\geq 18 \mu \mathrm{g} / 1$ in all children) and free thyroxine (FT4). After 12 months of GHT, in 7 children who showed basal cortisol levels $<18 \mu \mathrm{g} / \mathrm{l}$ we performed a low-dose short adrenocorticotropin test, which showed a normal cortisol response.

As circulating insulin sensitivity markers, the serum levels of adiponectin and resistin after an overnight fast were also measured. The institutional Ethics Committee of the University of Palermo approved this study. At the time of hospitalization, an informed consent for the scientific use of the data was obtained from both the participants and their parents.

\subsection{Hormone and biochemical assays}

Glucose was measured in the centralized accredited laboratories of the University of Palermo with the standard methods. HbA1c levels were determined by HPLC with an ion-exchange resin (BioRad D10, BioRad, Milan, Italy). Serum insulin was measured by electrochemiluminescence (ECLIA, Elecsys Insulin, Roche, Milan, Italy). The sensitivity of the method was $0.4 \mu \mathrm{U} / \mathrm{ml}$. The normal range $(\mu \mathrm{U} / \mathrm{ml})$ was 2.6-24.9. Serum GH levels were measured by Immunoassay in electrochemiluminescence (ECLIA, Elecsys hGH, Roche, Milan, Italy). The lower limit of detection of the assay was $0.030 \mu \mathrm{g} / \mathrm{l}$. The intra- and inter-assay coefficients of variation (CV) were $0.6-5.0$ and $3.8-5.0 \%$, respectively. We reported GH concentrations in $\mu \mathrm{g} / 1$ of IS $98 / 574$. Serum IGF-I levels were measured by means of a chemiluminescent immunometric assay (Immulite 2000; Diagnostic Products Corp., Los Angeles, CA) using murine monoclonal anti-IGF-I antibodies. The standards were calibrated against the World Health Organization second IS $87 / 518$. The sensitivity was $1.9 \mu \mathrm{g} / \mathrm{l}$. The intra- and interassay CVs were $2.3-3.9 \%$ and $3.7-8.1 \%$, respectively. Adiponectin ( $\mu \mathrm{g} /$ $\mathrm{ml})$ and resistin $(\mathrm{ng} / \mathrm{ml})$ were assayed using an ELISA sandwich enzyme immuno-assay (BioVendor, Heidelberg, Germany).

\subsection{Statistical analysis}

The Statistical Packages for Social Sciences SPSS version 19 was used for data analysis. Baseline characteristics were presented as mean \pm SD for continuous variables. Normality of distribution for quantitative variables was assessed with the Kolmogorov-Smirnov test. The differences between GHD and controls were analyzed by Student's $t$-test, except for GH peak after glucagon and arginine test and fasting insulin levels, which were analyzed by Mann-Whitney $U$ test (nonparametric test), as they were continuous variables without normal distribution. The differences between paired continuous variables in the GHD group (before and 12 months after GHT) were analyzed using the paired Student's $t$-test for continuous variables, except for fasting insulin levels, which were analyzed by the Wilcoxon test. Pearson's correlation was performed among continuous variables. A $p$ value $<0.05$ was considered statistically significant.

\section{Results}

\subsection{Clinical and hormonal parameters}

The clinical and hormonal features of control subjects and GHD 
Table 1

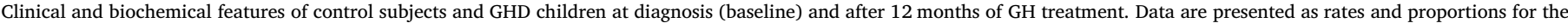
categorical data and as mean \pm standard deviation (SD) for the continuous variables.

\begin{tabular}{|c|c|c|c|c|c|}
\hline & Control group (N. 12) & GHD at baseline (N. 23) & GHD at 12 months (N. 23) & $p$ & $p^{*}$ \\
\hline Gender & & & & 0.483 & - \\
\hline Males & $7(58)$ & $15(65)$ & 15 (65) & & \\
\hline \multirow[t]{2}{*}{ Females } & $5(42)$ & $8(35)$ & $8(35)$ & & \\
\hline & Mean $\pm S D$ & Mean $\pm S D$ & Mean $\pm S D$ & & \\
\hline Age (years) & $7.8 \pm 0.9$ & $8.4 \pm 1.0$ & - & 0.086 & - \\
\hline Height (SD) & $-2.1 \pm 0.1$ & $-2.2 \pm 0.2$ & $-1.8 \pm 0.1$ & 0.072 & $<0.001$ \\
\hline Growth velocity (cm/year) & $4.7 \pm 0.9$ & $3.5 \pm 0.8$ & $7.7 \pm 1.8$ & $<0.001$ & $<0.001$ \\
\hline BMI $\left(\mathrm{kg} / \mathrm{m}^{2}\right)$ & $17.1 \pm 2.8$ & $17.2 \pm 2.3$ & $17.6 \pm 2.5$ & 0.939 & 0.306 \\
\hline $\mathrm{WC}(\mathrm{cm})$ & $64.2 \pm 10.7$ & $62.5 \pm 10.2$ & $63.7 \pm 9.3$ & 0.640 & 0.086 \\
\hline IGF-I (SD) & $1.1 \pm 0.4$ & $-0.7 \pm 0.2$ & $1.0 \pm 0.3$ & $<0.001$ & $<0.001$ \\
\hline GH peak after glucagon test $(\mu \mathrm{g} / \mathrm{l})$ & $11.1 \pm 1.7$ & $4.4 \pm 1.7$ & - & $<0.001$ & - \\
\hline GH peak after arginine test $(\mu \mathrm{g} / \mathrm{l})$ & $16.6 \pm 6.0$ & $4.1 \pm 2.0$ & - & $<0.001$ & - \\
\hline Fasting glucose (mmol/L) & $78.9 \pm 3.3$ & $82.9 \pm 9.0$ & $87.1 \pm 11.9$ & 0.067 & 0.101 \\
\hline HbA1c (\%) & $5.1 \pm 0.3$ & $5.2 \pm 0.2$ & $5.3 \pm 0.3$ & 0.713 & 0.053 \\
\hline Fasting insulin (IU/ml) & $3.3 \pm 2.0$ & $4.2 \pm 2.0$ & $9.5 \pm 5.0$ & 0.220 & $<0.001$ \\
\hline ISI-Matsuda & $12.3 \pm 3.7$ & $11 \pm 3.6$ & $5 \pm 1.8$ & 0.343 & $<0.001$ \\
\hline M-value $(\mathrm{mg} / \mathrm{kg} / \mathrm{min})$ & $4.5 \pm 0.5$ & $4.8 \pm 0.5$ & $3.4 \pm 0.7$ & 0.064 & $<0.001$ \\
\hline Adiponectin $(\mu \mathrm{g} / \mathrm{ml})$ & $12.8 \pm 3.5$ & $11.6 \pm 2.3$ & $11.8 \pm 2.6$ & 0.219 & 0.649 \\
\hline Resistin (ng/ml) & $3.2 \pm 0.8$ & $3.9 \pm 1.1$ & $4.6 \pm 2.1$ & 0.274 & 0.028 \\
\hline
\end{tabular}

$p=$ difference between control group and GHD subjects at baseline.

$p^{*}=$ difference between GHD subjects at baseline and GHD subjects at 12 months of GH treatment.

children at diagnosis and after 12 months of GHT are shown in Table 1.

At baseline, no significant difference in height, BMI and WC between GHD children and controls was found. As expected, GHD children showed significantly lower growth velocity $(3.5 \pm 0.8$ vs. $4.7 \pm$ $0.9 \mathrm{~cm} ; \quad p<0.001)$, IGF-I $(-0.7 \pm 0.2$ vs. $1.1 \pm 0.4 \mathrm{SDl}$; $p<0.001)$ and GH peak after both glucagon (4.4 \pm 1.7 vs. $11.1 \pm 1.7 \mu \mathrm{g} / \mathrm{l} ; \quad p<0.004)$ and arginine test $(4.1 \pm 2.0$ vs. $16.6 \pm 6.0 \mu \mathrm{g} / \mathrm{l} ; p<0.001$ ) than control subjects.

In GHD children after 12 months we observed a significant increase in growth (height: $-1.8 \pm 0.1$ vs. $-2.2 \pm 0.2$ SDS; $p<0.001$; growth velocity: $7.7 \pm 1.8$ vs. $3.5 \pm 0.8 \mathrm{~cm} ; p<0.001$ ), with a concomitant increase in IGF-I $(1.0 \pm 0.3$ vs. $-0.7 \pm 0.2 \mathrm{SD}$; $p<0.001$ ), while no significant change in BMI and WC was found (Table 1).

\subsection{Metabolic parameters}

The metabolic parameters of control subjects and GHD children at diagnosis and after 12 months of GHT are shown in Table 1.

At baseline, no significant difference was found in fasting glucose, insulin and HbA1c between GHD children and controls. Similarly, no significant difference was found in ISI Matsuda, M-value (Fig. 1) adiponectin and resistin levels between the 2 groups (Table 1).

After 12 months of GHT, GHD children showed a significant increase in fasting insulin $(9.5 \pm 5.0$ vs. $4.2 \pm 2.0 \mathrm{I} / \mathrm{ml} ; p<0.001)$, with a concomitant significant decrease in ISI Matsuda $(5.0 \pm 1.8$ vs. $11 \pm 3.6 ; p<0.001)$ and M-value (3.7 \pm 0.7 vs. $4.8 \pm 0.5 \mathrm{mg} / \mathrm{kg} /$ min; $p<0.001$ ) (Fig. 1), without significant change in fasting glucose, and HbA1c (Table 1). Adiponectin levels did not show any significant change after GHT $(11.8 \pm 2.6$ vs. $11.6 \pm 2.3 \mu \mathrm{g} / \mathrm{ml} ; p=0.649)$, while a significant increase in resistin levels $(4.6 \pm 2.1$ vs. $3.9 \pm 1.1 \mathrm{ng} / \mathrm{ml} ; p=0.028$ ) was found.

At baseline, GHD children showed a significant and weak correlation between M-value and ISI Matsuda (rho 0.418, $p=0.047$ ), while no correlation was found between M-value and BMI, WC, adiponectin, resistin and IGF-I. After 12 months of GHT, the M-value did not show any significant correlation with any other metabolic parameter analyzed (Table 2). The additional analysis evaluating the correlation between the delta (change from baseline to 12 months) of the parameters evaluated in the model did not show any significant correlation between the delta of M-value and that of the dependent variables (data

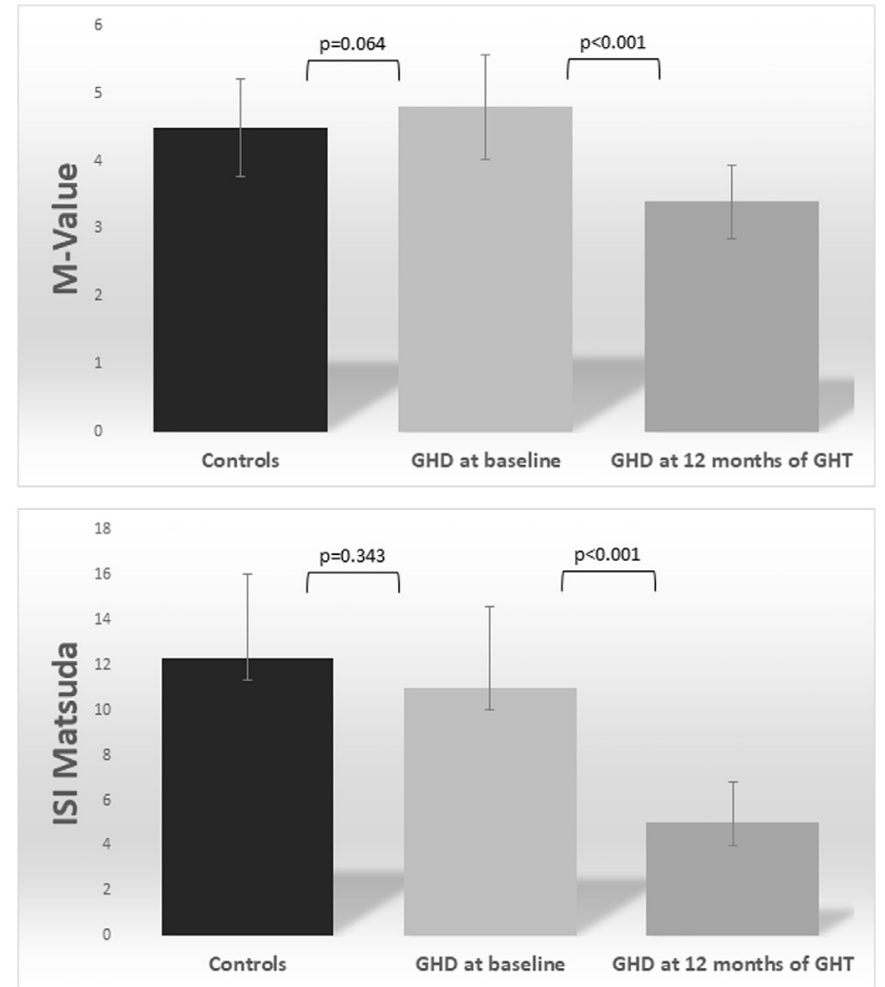

Fig. 1. M-value (clamp-derived) and ISI-Matsuda of control subjects and GHD children at diagnosis and after 12 months of GH treatment.

not shown). No correlation was found among ISI Matsuda, adiponectin and resistin levels (data not shown).

\section{Discussion}

In this study we found that 12 months of GHT lead to a deterioration in insulin sensitivity, although the glucose tolerance seems to be unaffected, and that the euglycemic hyperinsulinemic clamp, considered as the gold standard technique to assess insulin sensitivity, does not correlate with the other surrogate indices routinely used in clinical 
Table 2

Correlation (univariate analysis) between M-value derived by euglycemic hyperinsulinemic clamp and hormonal and metabolic parameters at baseline and after 12 months of GH treatment in GHD children.

Independent variables Dependent variable: M-value (clamp-derived)

\begin{tabular}{llllll}
\cline { 5 - 6 } & \multicolumn{3}{l}{ Baseline } & & \multicolumn{2}{l}{12 months } \\
\cline { 2 - 3 } \cline { 5 - 6 } \cline { 5 - 6 } & $r$ & $p$ & & $r$ & $p$ \\
\hline IGF-I (SD) & -0.138 & 0.529 & & -0.112 & 0.611 \\
BMI $\left(\mathrm{kg} / \mathrm{m}^{2}\right)$ & -0.137 & 0.534 & & -0.005 & 0.983 \\
WC $(\mathrm{cm})$ & -0.026 & 0.906 & & -0.093 & 0.673 \\
ISI Matsuda & 0.418 & 0.047 & & 0.052 & 0.813 \\
Adiponectin $(\mu \mathrm{gg} / \mathrm{ml})$ & 0.032 & 0.884 & & 0.264 & 0.223 \\
Resistin $(\mathrm{ng} / \mathrm{ml})$ & -0.274 & 0.205 & & 0.073 & 0.740 \\
\hline
\end{tabular}

\section{practice.}

It is known that $\mathrm{GH}$, in addition to its auxological effects, plays a key metabolic role [1]. If these metabolic effects are frequently unnoticed in the pediatric age, probably due to the short duration of GHD compared with adult patients, they occur more often during GHT. Indeed, in our cohort of patients at baseline no significant difference was found in all metabolic parameters analyzed.

Increased insulin resistance, through impaired suppression of glucose production and impaired stimulation of glucose utilization, has been demonstrated after continuous GH infusion [8]. GHT is associated with an increase in insulin levels, although whether GH-induced hyperinsulinemia should be considered an index of relative insulin resistance is not clear. Indeed, a deterioration of the basal indices of insulin resistance, like Homa-IR, is widely demonstrated by many studies performed in GHD children [9-11].

Conversely, based on Homa-IR values, Capalbo et al. recently reported that GHT is not associated with significant impairment of insulin sensitivity in GHD children [12]. These discordant data are probably due to the inability of Homa-IR to reliably assess insulin sensitivity. Indeed, the increase in Homa-IR may just represent an expected consequence of GH-induced basal hyperinsulinemia and may lead to discordant results. These data are confirmed by the current study. Indeed, we found a significant increase in fasting insulin after 12 months of GHT, even without evident changes in glucose tolerance, as demonstrated by the unchanged glucose and HbA1c levels.

Certainly ISI Matsuda, which derives from glucose and insulin levels during OGTT, shows a different behavior and represents a more reliable index of insulin sensitivity than the basal Homa-IR [13]. In this study, as expected, we found a significant decrease in ISI Matsuda after GHT. However, available data about a relationship between ISI Matsuda and other indices of insulin sensitivity, such as the gold standard clamp [4] or with adipokine levels [14], are scarce and do not seem to confirm the full reliability of this index.

In this study we aimed to assess the performance of both ISI Matsuda and the gold standard clamp. These data suggest that ISI Matsuda may be useful to detect changes in insulin sensitivity, although a weak correlation to M-value (at baseline) and no correlation (after 12 months of GHT) were reported. In our opinion, probably the small number of sample may be the cause of the lack of correlation between the 2 indices although, in this connection, comparative data about the insulin sensitivity degree in GHD children assessed by these 2 indices are unavailable from literature.

In a large group of childhood cancer survivors with GHD in whom insulin sensitivity was measured by euglycemic hyperinsulinemic clamp, Petryk et al. showed lower M value than controls [15], while in the current study we did not find any significant difference in M-value between GHD and controls, probably due to the major difference in patients evaluated. A significant impact of GHT on M-value has been reported by Norrelund et al., who showed an increase in insulin sensitivity after 12 months of discontinuation of GHT in 18 GHD patients during transition from childhood to adulthood, with a subsequent deterioration when GHT was restarted [16].

We have previously reported similar results in a small group of prepubertal GHD children, in whom we showed a significant deterioration in M-value after 12 months of GHT [4]. The current study, performed in a larger cohort of patients, confirms these data. Indeed, a significant decrease in insulin sensitivity assessed by clamp was documented after GHT. The mechanisms of the deterioration of insulin sensitivity during GHT are probably multiple and related to the counter-regulatory action of the GH, since neither IGF-I nor BMI or WC individually showed a significant correlation with M-value.

Circulating plasma markers of insulin sensitivity, such as some adipokines, have been tested in GHD children. Adiponectin levels are recognized to be inversely related to insulin resistance [17]. GH has been shown to decrease the expression of adiponectin in cultured human adipose tissue [18]. However, data about the impact of GHT on adiponectin are quite controversial. Adiponectin seems to be unaffected in untreated GHD [19] and GHT seems to have discordant effects on adiponectin, leaving it unchanged or only slightly modifying it $[11,19-22]$. Conversely, an increase in adiponectin has been documented in GHD children by other studies $[23,24]$. The available data about the relationship between adiponectin levels and insulin sensitivity indices are very scarce. We found a lack of correlation between insulin sensitivity and change in adiponectin levels, in agreement with previous studies. Indeed, Hana et al. showed that GHT in adult GHD is effective in changing body composition but without concomitant changes in adiponectin levels [20]. Similarly, we already demonstrated that the change in insulin sensitivity during GHT in children, assessed with both Homa-IR and ISI Matsuda, is not concomitant with change in adiponectin levels $[11,14]$ and similar results have been obtained by other studies [24].

Resistin has been shown to be linked to insulin resistance [25], but the few available data about the impact of GHD or GHT on its circulating levels are quite controversial. No difference in resistin levels between adult GHD patients and controls was reported by Salman et al. [26] and our current data are in agreement with that study. Indeed, resistin levels showed no significant difference between GHD children at baseline and controls. Although marked and sustained up-regulation of resistin by GH has been demonstrated [27]. Meazza et al. showed higher resistin levels in 17 GHD children and a subsequent decrease in these levels after 12 months of GHT [28], while other studies showed that GHT does not seem able to significantly modify resistin levels in adult GHD patients [26].

In a previous report we showed a trend to an increase in resistin, though not statistically significant, in children during GHT [14]. Similarly, Nozue et al. reported a rise in resistin after 1 months of GHT in 20 children, regardless of change in other metabolic parameters [29] and our study confirms this finding, showing a rise in resistin after GHT.

These controversial data about adipokine levels indicate that the change in them are probably not really correlated with the change in insulin sensitivity degree, as demonstrated by the lack of correlation among adipokine levels and M-value shown in the current study. Indeed, if at baseline we found that clamp showed a correlation, even fairly weak, only with ISI Matsuda and not with adipokine levels, after 12 months of GHT the adipokine levels did not show a correlation with clamp. In our opinion a number of factors, first of all the fat mass, may influence adipokine levels and make them unreliable markers, since the amount of fat mass is not necessarily correlated with the quality of fat.

A limitation of this study may certainly be related to the small size of the population. Indeed, we believe that these data must be validated in additional larger prospective studies. However, clamp represents an invasive and expensive procedure and therefore it is difficult to apply routinely in clinical practice, especially in children. Other limitations may be represented by the short-term follow-up and the lack of data of the control group after 12 months of follow-up. Although the main metabolic effects of GHT generally occur during the first months of 
treatment, a longer follow-up may be useful to have more reliable data, both in GHD children and control subjects. In addition, adding data on body composition evaluated by dual energy X-ray absorptiometry (DEXA) will certainly confirm these important data in future studies.

Conversely, the strength of this study lies in the fact that for the first time it simultaneously evaluated the clamp with other indices and plasma markers of insulin sensitivity in GHD children.

In conclusion, this study highlights the limit of the evaluation of insulin sensitivity performed through surrogate indices or circulating markers, such as adipokines, which may lead to controversial data and do not correlate with the gold standard technique to assess insulin sensitivity.

\section{Disclosure statement}

The authors have nothing to disclose.

\section{Grants}

This research did not receive any specific grant from any funding agency in the public, commercial or non-profit sector.

\section{Conflict of interest}

All authors declare that there is no conflict of interest that could be perceived as prejudicing the impartiality of the research reported.

\section{References}

[1] A. Vijayakumar, R. Novosyadlyy, Y. Wu, et al., Biological effects of growth hormone on carbohydrate and lipid metabolism, Growth Hormon. IGF Res. 20 (2010) 1-7.

[2] B. Schwartz, D.R. Jacobs Jr., A. Moran, J. Steinberger, C.P. Hong, A.R. Sinaiko, Measurement of insulin sensitivity in children: comparison between the euglycemic-hyperinsulinemic clamp and surrogate measures, Diabetes Care 31 (4) (2008) 783-788.

[3] R.A. DeFronzo, J.D. Tobin, R. Andres, Glucose clamp technique: a method for quantifying insulin secretion and resistance, Am. J. Phys. 237 (3) (1979) E214-223.

[4] A. Ciresi, M.C. Amato, C. Giordano, Reduction in insulin sensitivity and inadequate B-cell capacity to counteract the increase in insulin resistance in children with idiopathic growth hormone deficiency during 12 months of growth hormone treatment, J. Endocrinol. Investig. 38 (2015) 351-359.

[5] Growth Hormone Research Society, Consensus guidelines for the diagnosis and treatment of growth hormone (GH) deficiency in childhood and adolescence: summary statement of the GH Research Society, J. Clin. Endocrinol. Metab. 85 (2000) 3990-3993.

[6] W. Greulich, S. Pyle, Radiographic Atlas of Skeletal Development of the Hand and Wrist, 2nd ed, Stanford University Press, Stanford, CA, 1959.

[7] M. Matsuda, R.A. DeFronzo, Insulin sensitivity indices obtained from oral glucose tolerance testing: comparison with the euglycemic insulin clamp, Diabetes Care 22 (1999) 1462-1470.

[8] R.A. Rizza, L.J. Mandarino, J.E. Gerich, Effects of growth hormone on insulin action in man. Mechanisms of insulin resistance, impaired suppression of glucose production, and impaired stimulation of glucose utilization, Diabetes 31 (8 Pt 1) (1982) 663-669.

[9] R.A. Heptulla, S.D. Boulware, S. Caprio, et al., Decreased insulin sensitivity and compensatory hyperinsulinemia after hormone treatment in children with short stature, J. Clin. Endocrinol. Metab. 82 (1997) 3234-3238.
[10] P. Saenger, Metabolic consequences of growth hormone treatment in paediatric practice, Horm. Res. 53 (2000) 60-69.

[11] A. Ciresi, M.C. Amato, A. Criscimanna, et al., Metabolic parameters and adipokine profile during GH replacement therapy in children with GH deficiency, Eur. J. Endocrinol. 156 (2007) 353-360.

[12] D. Capalbo, A. Esposito, N. Improda, et al., Glucose homeostasis in GHD children during long-term replacement therapy: a case-control study, Endocrine (2017), http://dx.doi.org/10.1007/s12020-017-1408-0 (Epub ahead of print).

[13] A. Ciresi, F. Cicciò, S. Radellini, V. Guarnotta, A.M. Calcaterra, C. Giordano, More favorable metabolic impact of three-times-weekly versus daily growth hormone treatment in Naïve GH-deficient children, Int. J. Endocrinol. 2017 (2017) 8469680.

[14] A. Ciresi, G. Pizzolanti, M. Leotta, V. Guarnotta, G. Teresi, C. Giordano, C, Resistin, visfatin, leptin and omentin are differently related to hormonal and metabolic parameters in growth hormone-deficient children, J. Endocrinol. Investig. 39 (9) (2016) 1023-1030.

[15] A. Petryk, K.S. Baker, B. Frohnert, et al., Blunted response to a growth hormone stimulation test is associated with unfavorable cardiovascular risk factor profile in childhood cancer survivors, Pediatr. Blood Cancer 60 (3) (2013) 467-473.

[16] H. Nørrelund, N. Vahl, A. Juul, et al., Continuation of growth hormone (GH) therapy in GH-deficient patients during transition from childhood to adulthood: impact on insulin sensitivity and substrate metabolism, J. Clin. Endocrinol. Metab. 85 (5) (2000) 1912-1917.

[17] C. Weyer, T. Funahashi, S. Tanaka, et al., Hypoadiponectinemia in obesity and type 2 diabetes: close association with insulin resistance and hyperinsulinemia, J. Clin. Endocrinol. Metab. 86 (2001) 1930-1935.

[18] L. Nilsson, N. Binart, M. Bohlooly, et al., Prolactin and growth hormone regulate adiponectin secretion and receptor expression in adipose tissue, Biochem. Biophys. Res. Commun. 331 (4) (2005) 1120-1126.

[19] J. Svensson, H. Herlitz, P.A. Lundberg, G. Johannsson, Adiponectin, leptin, and erythrocyte sodium/lithium countertransport activity, but not resistin, are related to glucose metabolism in growth hormone-deficient adults, J. Clin. Endocrinol. Metab. 90 (2005) 2290-2296.

[20] V. Hana, J.V. Silha, V. Justova, Z. Lacinova, J.J. Stepan, L.J. Murphy, The effects of GH replacement in adult GH-deficient patients: changes in body composition without concomitant changes in the adipokines and insulin resistance, Clin. Endocrinol. 60 (4) (2004) 442-450.

[21] R.H. Willemsen, M. van Dijk, Y.B. de Rijke, A.W. van Toorenenbergen, P.G. Mulder, A.C. Hokken-Koelega, Effect of growth hormone therapy on serum adiponectin and resistin levels in short, small-for-gestational-age children and associations with cardiovascular risk parameters, J. Clin. Endocrinol. Metab. 92 (1) (2007) 117-123.

[22] B. Edén Engström, P. Burman, C. Holdstock, F.A. Karlsson, Effects of growth hormone (GH) on ghrelin, leptin, and adiponectin in GH-deficient patients, J. Clin. Endocrinol. Metab. 88 (11) (2003) 5193-5198.

[23] D. Capalbo, G. Mattace Raso, A. Esposito, et al., Cluster of cardiometabolic risk factors in children with GH deficiency: a prospective, case-control study, Clin. Endocrinol. 80 (2013) 856-862.

[24] J.P. López-Siguero, L.F. López-Canti, R. Espino, et al., Effect of recombinant growth hormone on leptin, adiponectin, resistin, interleukin-6, tumor necrosis factor- $\alpha$ and ghrelin levels in growth hormone-deficient children, J. Endocrinol. Investig. 34 (2011) 300-306.

[25] A.R. Shuldiner, R. Yang, D.W. Gong, Resistin, obesity, and insulin resistance-the emerging role of the adipocyte as an endocrine organ, N. Engl. J. Med. 345 (18) (2001) 1345-1346.

[26] S. Salman, A.K. Uzum, A. Telci, F. Alagol, N.C. Ozbey, Serum adipokines and low density lipoprotein subfraction profile in hypopituitary patients with growth hormone deficiency, Pituitary 15 (3) (2012) 386-392.

[27] P.J. Delhanty, D. Mesotten, F. McDougall, R.C. Baxter, Growth hormone rapidly induces resistin gene expression in white adipose tissue of spontaneous dwarf (SDR) rats, Endocrinology 143 (6) (2002) 2445-2448.

[28] C. Meazza, H.H. Elsedfy, S. Pagani, E. Bozzola, M. El Kholy, M. Bozzola, Metabolic parameters and adipokine profile in growth hormone deficient (GHD) children before and after 12-month GH treatment, Horm. Metab. Res. 46 (3) (2014) 219-223.

[29] H. Nozue, T. Kamoda, A. Matsui, Serum resistin concentrations in growth hormonedeficient children during growth hormone replacement therapy, Metabolism 56 (11) (2007) 1514-1517. 